\title{
Frigatebirds on Aldabra Atoll: population census, recommended monitoring protocol and sustainable tourism guidelines
}

\author{
MICHAL ŠÚR, NANCY BUNBURY and \\ JANSKE VAN DE CROMMENACKER
}

\section{Summary}

We report the results of a survey of breeding Greater Frigatebird Fregata minor and Lesser Frigatebird F. ariel on Aldabra Atoll, a UNESCO World Heritage site in the Seychelles archipelago, which hosts the largest breeding population of frigatebirds in the Indian Ocean. All four colonies across the atoll were surveyed in 2011 and 2012 by counting adults on nests and chicks, and calculating the number of fledged birds. The breeding population in 2011 consisted of approximately 4,400 pairs of Greater Frigatebird and 6,60o pairs of Lesser Frigatebird. This is a potential increase of at least $10 \%$ since the last comparable surveys in 1976/77 and 2000. However, 2012 populations were considerably less than in 2011 , highlighting significant annual variation and the need for more data. We also report a new breeding colony on the island of Picard, which was last reported to host breeding frigatebirds a century ago. We outline recommendations for future frigatebird monitoring on Aldabra and elsewhere and revisions to tourist regulations resulting from the census.

\section{Introduction}

Seabirds are recognised as important regional and global indicators of marine system functioning yet they are also the most threatened avian group, with $28 \%$ of species considered to be globally threatened and nearly half known or suspected to be experiencing population declines (Croxall et al. 2012). Monitoring of key seabird populations, and subsequent conservation measures, are therefore critical components of Marine Protected Area management.

Aldabra Atoll, a UNESCO World Heritage site, Important Bird Area and Marine Protected Area, is a large raised coral atoll in the south-western Indian Ocean (Figure 1). Aldabra hosts numerous breeding seabird populations, including the largest frigatebird colony in the Indian Ocean (Burger and Betts 2001). On Aldabra, Greater Frigatebirds Fregata minor, and Lesser Frigatebirds, F. ariel, nest together in mixed colonies (Diamond 1975, Orta 1992) although both species share a mainly Indo-Pacific distribution. For the few tourists that visit Aldabra, the atoll's frigatebirds are one of its main attractions. As frigatebirds are sensitive to disturbance, a set of guidelines is implemented to enable visitor viewing with minimal disturbance to the colonies.

Aldabra's frigatebirds have been surveyed several times using different methods (Diamond 1975, Reville 1980, 1983, Burger and Betts 2001, Weimerskirch et al. 2010). An extensive annual breeding frigatebird survey conducted in the late 1970s estimated at least 4,000 pairs of Greater and 6,00o pairs of Lesser Frigatebird (Reville 1980). The most recent atoll-wide survey, carried out in 2000 (Burger and Betts 2001) was less comprehensive, but showed that numbers and species composition were similar to Reville's survey. During a survey in 2006 conducted solely at the largest colony of Middle Camp (Weimerskirch et al. 2010), considerably more active nests 


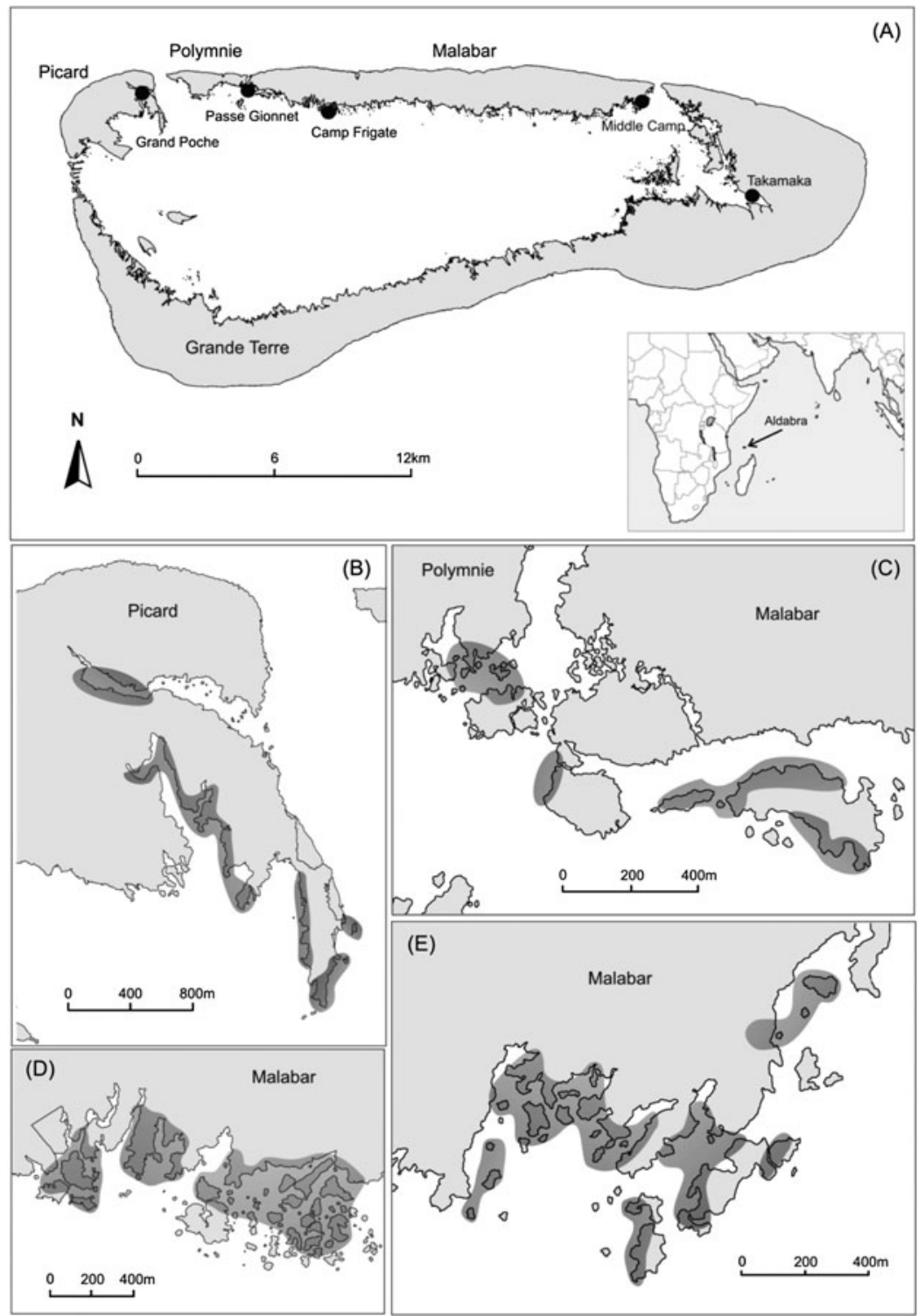

Figure 1. (A) Location of Aldabra Atoll in the Indian Ocean (inset) with locations of current and historical Greater and Lesser Frigatebird colonies surveyed in 2011 and 2012, and individual colonies Grand Poche (B), Passe Gionnet (C), Camp Frigate (D), and Middle Camp (E).

were found than in previous studies: 2.4 times and 3.6 times more nests than found by Reville (1980) and Burger and Betts (2001), respectively. However, in addition to the large gaps between surveys, each previous study applied different methods and was conducted at a different time of year, so the results are not directly comparable. The management of this important seabird population therefore requires a logistically feasible and repeatable survey method that can be used routinely to monitor and respond to population trends. 
We report the results of a survey of breeding Greater and Lesser Frigatebirds carried out over two seasons on Aldabra. The primary aim of the research is to update information on the current breeding population size of both species on Aldabra. We further aimed to design a method that is practicable and easily replicable. Based on our data on colony size and distribution, we outline recommendations for monitoring frigatebirds breeding on Aldabra that can also be used in other key colonies of these seabirds.

\section{Methods}

\section{Study area}

The survey was carried out on Aldabra Atoll $\left(9^{\circ} 24^{\prime} \mathrm{S}, 46^{\circ} 20^{\prime} \mathrm{E}\right)$, in the south-west Indian Ocean (Figure 1). Aldabra is a large $(34 \times 14.5 \mathrm{~km})$ raised coralline atoll consisting of a rim of four main islands with a total land surface area of approximately $155 \mathrm{~km}^{2}$ surrounding a large lagoon c.30 km across (Stoddart et al. 1971). Aldabra was inscribed on the UNESCO World Heritage list in 1982 and has been managed entirely for research and conservation since 1979 by the Seychelles Islands Foundation (SIF).

Aldabra's frigatebirds nest on the inside of the lagoon in mangrove habitat. Greater Frigatebirds nest exclusively in mangroves, especially Rhizophora mucronata and Bruguiera gymnorhiza, while Lesser Frigatebirds nest in Pemphis acidula and other shrubs, as well as in mangroves (Reville 1980). Frigatebirds have recently been recorded nesting only on the north side of the lagoon in four separate colonies (Grand Poche, Passe Gionnet, Camp Frigate and Middle Camp; Figure 1), which were all covered in our survey. In the 196os another colony was active at Bras Takamaka (Figure 1; Diamond 1975), but breeding has not occurred there since the 1970 and we confirmed that there were no nesting frigatebirds at this location in either of our study years. Other parts of the atoll are visited regularly and there are no other active colonies at present.

\section{Survey methods}

Due to the lengthy frigatebird breeding cycle (12-19 months from laying to fledging), individuals do not breed annually; however, seasonal peaks in breeding activity are seen (Reville 1980, 1983). On Aldabra, the main laying period is reported as Jun-Sept for lesser and Aug-Oct for Greater Frigatebirds (Reville 1980, 1983). Our survey was conducted in two consecutive years at the beginning of the calendar year (Jan-Feb 2011 and Jan 2012) to minimise disturbance to breeding birds since at this time most chicks are relatively large, and fewer unpaired males are present to threaten unattended nests (e.g. by stealing nest material from disturbed birds). Furthermore, incubating/brooding adults were still present at the nests, providing additional information on species identification and composition.

The survey was carried out by a team of four (three counters, one skipper) from a 16 ' fibreglass boat with a $25 \mathrm{hp}$ engine. Due to logistical constraints it was not possible to follow earlier recommendations to use kayaks or punters (Reville 1980, Burger and Betts 2001). The boat was driven slowly through the colony area at high tide, stopping when necessary. A distance of at least $15 \mathrm{~m}$ was maintained from the nesting birds at all times and noise was kept to a minimum. A small part of one colony (Middle Camp) was surveyed on foot at spring low tide. The precautions taken to avoid disturbance were successful as no birds were seen flying up from their nest as a result of our presence. The extent of each colony was mapped using GPS (Figure 1 ).

The total number of active nests was assessed, with each of the three counters focusing on one of the following categories: 1) Lesser Frigatebird adult on nest (incubating/brooding small chick); 2) Greater Frigatebird adult on nest (incubating/brooding small chick); and 3) lone chick on nest (species not distinguished). Lone young birds on nests that had at least some down on the chest were counted as chicks. It was only possible to distinguish young chicks to species when attended by their parents, which required longer observation than was feasible given the limited active 
survey time. Adult flying birds, roosting birds and independent juveniles were excluded from the census. The few displaying birds observed were also excluded, as their inclusion would increase the risk of double counting due to their mobility and the lack of knowledge concerning their pair-bond associations. The number of dependent fledged juveniles was calculated based on Reville's figures (1980). All counters were trained and tested in species identification by the team leader (MŠ) and had practice sessions in a colony before the survey began.

\section{Data analysis}

As our count consisted partly of chicks that could not be differentiated to species, we used adult species ratio across individual colonies as a proxy for chick species composition (Table IA). Furthermore, the breeding population includes adults feeding fledged juveniles, which depend on parental care for 4-12 months after fledging (Reville 1980), but these are problematic to count accurately, since they spend only $26-50 \%$ of their time at the colony (Diamond 1975). Their number was therefore calculated based on a previous estimate of $37 \%$ Greater and $11 \%$ Lesser Frigatebird pairs feeding fledglings (derived from Reville 1983; Table 1A).

\section{Results}

We calculated the total breeding population of frigatebirds on Aldabra to be 9,891 (4,198 Greater, 5,693 Lesser) pairs in 2011, and 5,708 (2,654 Greater, 3,054 Lesser) pairs in 2012 (Table 1A). Figures for both seasons are conservative and we estimate the breeding population size to have been at least 11,000 (4,400 Greater, 6,600 Lesser) pairs in 2011, and at least 6,500 (3,000 Greater, 3,500 Lesser) pairs in 2012 for reasons discussed below.

The spatial distribution of colonies in both survey years was similar (Figure 1). A few small islets in the Camp Frigate colony which had no nests in 2011 hosted small groups of mainly Lesser Frigatebirds in 2012. The number of nests in 2012 was lower than in $2011(-42 \%)$, which was most apparent in the number of chicks (-63\%; Table $1 \mathrm{~A})$. This was most marked in the Camp Frigate colony (1,160 and 235 chicks in 2011 and 2012 respectively; $-80 \%$; Table $1 B$ ); compared to 2011 , the Lesser Frigatebirds in this colony were at early nesting stages (egg, young chick), while most Greater Frigatebirds had more developed chicks.

\section{Discussion}

Our census of the Aldabra frigatebird population indicated a minimum of 4,198 Greater and 5,693 Lesser Frigatebird pairs (Table IA). These figures are conservative estimates and the actual population size as derived from the 2011 survey is almost certainly higher (11,0oo pairs in total; 4,400 Greater, 6,60o Lesser), since birds hidden by vegetation are likely to have been missed. Even the higher figure is probably an underestimate since Reville's estimate of 10,000 pairs is based on counts during the breeding peak, whereas our surveys were at least three months later in the season. Pairs that failed early in the breeding season will have been missed by our survey. The likelihood that this bias occurred is high, since breeding success of frigatebirds on Aldabra is naturally low and rate of nest desertion is high (Weimerskirch et al. 2010).

Our observations of Lesser Frigatebirds incubating in January suggest that breeding seasonality in this species is less fixed than described by Reville (1980), possibly showing a year-round pattern. This potential change needs to be confirmed with further field research. Breeding seasonality of Greater Frigatebirds did correspond with Reville's reports, as we found very few incubating/brooding adults and most chicks were in an advanced stage.

The change in the breeding population size in 2012, mainly caused by a lower number of chicks, could reflect normal fluctuations in annual numbers of nesting frigatebirds on Aldabra and elsewhere. Frigatebirds feed on flying fish and squid associated with sub-surface predators, such 
Table 1. Results of 2011 and 2012 surveys of Greater Fregata minor and Lesser F. ariel Frigatebirds on Aldabra: (A) minimum total breeding population size calculated using three categories of individuals: adult birds on nest ('Adults'), lone chicks ('Chicks'; see Methods), and adults feeding dependent fledglings ('Feeding'; see Methods); and (B) comparison of number of nests within individual colonies.

\begin{tabular}{|c|c|c|c|c|c|c|c|c|c|c|}
\hline & & \multicolumn{4}{|l|}{2011} & \multicolumn{4}{|l|}{2012} & \multirow[t]{2}{*}{$\%$ change } \\
\hline & & Greater & Lesser & Chicks & Total & Greater & Lesser & Chicks & Total & \\
\hline \multirow[t]{4}{*}{ (A) Total population } & Adults & 370 & 3,034 & & $3,404(34.4 \%)$ & 384 & 2,498 & & $2,882(50.5 \%)$ & $-15 \%$ \\
\hline & Chicks & 2,367 & 2,005 & & $4,372(44.2 \%)$ & 1,427 & 179 & & $1,606(28.1 \%)$ & $-63 \%$ \\
\hline & Feeding & 1,461 & 654 & & $2,115(21.4 \%)$ & 843 & 377 & & $1,220(21.4 \%)$ & $-42 \%$ \\
\hline & Total & 4,198 & 5,693 & & 9,891 & 2,654 & 3,054 & & 5,708 & $-42 \%$ \\
\hline \multirow[t]{5}{*}{ (B) Individual colonies } & Grand Poche & 136 & 520 & 779 & $1,435(18.4 \%)$ & 74 & 413 & 381 & $868(19.3 \%)$ & $-40 \%$ \\
\hline & Passe Gionnet & 15 & 6 & 498 & $519(6.7 \%)$ & 14 & 57 & 221 & $292(6.5 \%)$ & $-44 \%$ \\
\hline & Camp Frigate & 125 & 1,374 & 1,160 & $2,659(34.2 \%)$ & 151 & 1,521 & 235 & $1,907(42.5 \%)$ & $-28 \%$ \\
\hline & Middle Camp & 94 & 1,134 & 1,935 & $3,163(40.7 \%)$ & 145 & 507 & 769 & $1,421(31.7 \%)$ & $-55 \%$ \\
\hline & Total & 370 & 3,034 & 4,372 & 7776 & 384 & 2,498 & 1,606 & 4,488 & $-42 \%$ \\
\hline
\end{tabular}


as tuna. Such prey patches are highly unpredictable and frigatebirds are known to focus on areas with higher productivity on a regional scale. This could result in annual population fluctuations, particularly when considering the potential impacts of tuna fishing on this association. Further multi-year studies would be needed to confirm this. Alternatively, food availability in late 2011 at the onset of breeding may have been lower than usual, thereby impacting upon breeding. Potentially supporting this, a change in seabird nesting patterns was reported during the same period by seabird researchers on other islands within the region (e.g. Europa, M. Le Corre pers. comm.).

Our results also revealed several changes across the four colonies, both within the two years of this survey (Table $1 B$ ) and compared to previous surveys. The 2011 survey was the first to record Lesser Frigatebirds breeding in the Grand Poche area (Figure 1 ) and the first record of frigatebirds breeding on the island of Picard for over 100 years. A colony at Picard was noted in 1910 (Fryer 1910) and is thought to have disappeared by the 1960s due to human interference (Gaymer 1967). Greater Frigatebirds were first observed breeding again at Grand Poche in 2008 (C. Onezia, pers. comm.). The reasons for re-establishment of this colony are not known but could be due to various factors. The end of exploitation on Picard in the late 1960s, combined with the increased disturbance to the nearby Passe Gionnet colony from increased tourism (prior to piracy destabilising the tourism industry in the Seychelles outer islands; SIF, unpubl. data) potentially encouraged breeding birds to return to Picard. During visits accompanying tourists, we have observed that breeding frigatebirds are highly sensitive to disturbance, leaving their nests if approached too closely or exposed to excessive mechanical or human noise. It is thus possible that some of the Passe Gionnet colony may have shifted to Grand Poche and subsequently attracted additional birds. Yet, other factors are also likely to have played a role, since the abandonment of the Bras Takamaka colony cannot be explained by changes in tourist numbers, and exploitation of this colony would have been limited due to its location and inaccessibility.

Existing Seychelles Islands Foundation regulations for visiting the frigatebird colonies included: visits are only permitted to one of the colonies; a distance of at least $20 \mathrm{~m}$ from nesting or roosting birds should be kept; all boats should keep close to the SIF lead boat; all noise, including engines, should be limited; and no crew or visitors should leave the boats when in the colony. As a result of our observations and this survey, the regulations for visitors to Aldabra have been revised as a precautionary measure to further minimise disturbance. Frigatebird viewing is now restricted to parts of Grand Poche. Although a recently established colony, it is situated in a more open and wider waterway than the previously visited Passe Gionnet colony, making it possible to view nesting birds from an increased minimum distance of $30 \mathrm{~m}$ (previously $20 \mathrm{~m}$ ). In addition, visits are not permitted during the peak breeding season and visitors are encouraged to take close-up photographs of roosting birds outside the nesting colony. The revised guidelines, in combination with Aldabra's current protection status, will help to maintain a stable or increasing frigatebird population.

At a time when seabirds are declining globally (Croxall et al. 2012), it is encouraging that this key population of frigatebirds on Aldabra is stable and has potentially increased by at least $10 \%$ since the last comparable survey. The considerable fluctuations in our two survey years, and the lower numbers of in nesting birds counted in 2012, warrant continued research to monitor trends and fluctuations. Repeated annual surveys using the methods described here, and at the same time of year (Jan-Feb), are required to accurately assess the status of these dynamic seabird populations and harness their potential as an ecological indicator for this marine ecosystem. Our methods can be readily applied to other frigatebird populations and we recommend further surveys of other regional and international colonies to obtain a global picture of the status and trends of frigatebirds and their breeding sites.

\section{Acknowledgements}

This research was carried out by the Seychelles Islands Foundation as part of the national project "Strengthening Seychelles' protected area system through NGO management modalities" funded by the Global Environment Facility (GEF Project ID \# 3925) and implemented by the 
Seychelles Department of Environment. We would like to thank the following SIF staff for their contribution to this project: Frauke Fleischer-Dogley for her instrumental support from the project's inception and constructive input into the manuscript, Stan Denis and Bevil Narty for boat support, Andy Gouffé, Nella Victor and Martijn van Dinther for help with the fieldwork, Philip Haupt for help with GIS and mapping, Jock Currie, Jude Brice, other Aldabra staff, Wilna Accouche and other Head Office staff for logistical and administrative support.

\section{References}

Burger, A. E. and Betts, M. (2001) Monitoring populations of Red-footed boobies Sula sula and frigatebirds Fregata spp. breeding on Aldabra Atoll, Indian Ocean. B. Brit. Ornithol. Club 121: 236-246.

Croxall, J. P., Butchart, S. H. M., Lascelles, B., Stattersfield, A. J., Sullivan, B. and Symes, A. (2012) Seabird conservation status, threats and priority actions: a global assessment. Bird Conserv. Internatn. 22: $1-34$.

Diamond, A. W. (1975) Biology and behaviour of Frigatebirds Fregata spp. on Aldabra Atoll, Indian Ocean. Ibis 117: 302-323.

Fryer, J. C. F. (1910) The structure and formation of Aldabra and neighbouring islands - with notes on their flora and fauna. Trans. Linn. Soc. Lond. Zool. 14: $401-422$.

Gaymer, R. (1967) Observation on the birds of Aldabra in 1964 and 1965. Atoll Res. B. 118: 113-125.
Orta, J. (1992) Family Fregatidae (Frigatebirds). Pp. 362-374 in J. Del Hoyo, A. Elliott and J. Sargatal, eds. Handbook of the birds of the world - volume 1 . Barcelona, Spain: Lynx Edicions.

Reville, B. J. (1980) Spatial and temporal aspects of breeding in the frigatebirds Fregata minor and Fregata ariel. $\mathrm{PhD}$ thesis, University of Aberdeen, UK.

Reville, B. J. (1983) Numbers of nesting frigatebirds, Fregata minor and Fregata ariel, on Aldabra Atoll Nature Reserve, Seychelles. Biol. Conserv. 27: 59-76.

Stoddart, D. R. (1971) Settlement, development and conservation of Aldabra. Phil. Trans. Roy. Soc. Lond. B. 26o: 611-628.

Weimerskirch, H., Le Corre, M., Tew Kai, E. and Marsac, F. (2010) Foraging movements of great frigatebirds from Aldabra Island: Relationship with environmental variables and interactions with fisheries. Progr. Oceanography 86: 204-213.

\section{MICHAL ŠÚR, NANCY BUNBURY, JANSKE VAN DE CROMMENACKER* \\ Seychelles Islands Foundation, P.O. Box 853, Victoria, Mahé, Seychelles.}

*Author for correspondence; e-mail: aldabraresearch@gmail.com

Received 18 July 2012; revision accepted 27 September 2012; Published online 28 March 2013 